has been used to separate the protein release factor into two components (Scolnick, Tompkins, Caskey and Nirenberg, Proc. US Nat. Acad. Sci., 61, 768; 1968). One component, called Rl, will effect the hydrolysis of formylmethionyl-tRNA-bound to a ribosome in the presence of either of the triplets UAA or UAG. The second component, $\mathrm{R} 2$, will do the same thing in the presence of either UAA or UGA. These discoveries help to clarify one problem, but raise another. Previously, it had been difficult to imagine how one molecule (either $t$ RNA or protein) could recognize the three triplets, UAG, UAA and UGA, without recognizing the related triplet UGG, which codes for the aminoacid tryptophane. With two proteins each doing part of the job, this now seems clearer.

It is widely assumed that the codon used for chain termination in vivo is UAA, and that UAG and UGA are rarely, if ever, used. This belief stems from the observation that strains of $E$. coli can be made in which UAG is principally read as an amino-acid rather than as chain termination, and yet these strains appear quite healthy. If UAG were used frequently as a signal for chain termination, these strains would be expected to grow poorly. A similar argument holds for UGA. Strains in which UAA is largely read as an amino-acid have not, however, been isolated, and it is thus supposed that such a change would be lethal. UAA is therefore indispensable for chain termination. But why retain both UAG and UGA ? Loss of either Rl or R2 by mutation should not, on these arguments, be harmful, since either factor would cause termination at UAA. Perhaps UAG and UGA are sometimes, though infrequently, used to signal chain termination.

\section{RIBOSOME FUNCTION}

\section{Ribosome Dissociotion}

\section{from our Cell Biology Correspondent}

THE current model of protein synthesis in bacteria postulates the binding of $30 \mathrm{~S}$ ribosomal subunits with $m$ RNA, F-met $t$ RNA and initiation factors to form an initiation complex, followed by the binding of a $50 \mathrm{~S}$ subunit to form the $70 \mathrm{~S}$ ribosome which is active in translation. This implies a cyclical association and dissociation of the two ribosomal subunits at the beginning and end of translation, and the question arises whether there may be factors which are specifically required for the dissociation of the $70 \mathrm{~S}$ ribosome.

In the latest issue of Proc. US Nat. Acad. Sci. $(61,761 ; 1968)$, Subramanian, Ron and Davis report experiments which indicate that in $E$. coli there is a specific dissociation factor which stoichiometrically causes the release of $30 \mathrm{~S}$ subunits from the $70 \mathrm{~S}$ ribosome. The search for the factor was prompted by the observation that when protein synthesis is blocked in various ways - with actinomycin $D$, puromycin or by starving of a required amino-acid or a carbon source$70 S$ ribosomes accumulate although the concentration of the two ribosomal subunits remains constant. By extracting native $30 \mathrm{~S}$ subunits with $1.0 \mathrm{M} \mathrm{NH}_{4} \mathrm{Cl}$, they isolated a protein which causes a rapid dissociation of $70 \mathrm{~S}$ ribosomes. All the evidence suggests that this factor acts by a stoichiometric rather than a catalytic reaction and, because it works in the absence of metabolite, the dissociation is apparently independent of a source of energy. Its relation to the other initiation factors has still to be established, but Subramanian et al. have found that $\mathrm{Mg}^{++}$ions antagonize the response of the $70 \mathrm{~S}$ ribosome to the factor. This may imply that the factor, $70 S$ ribosomes, ribosome subunits and $\mathrm{Mg}^{++}$ions are all in equilibrium, which suggests a model in which the factor causes the dissociation of free $70 \mathrm{~S}$ ribosomes and is then displaced at the formation of new $70 S$ ribosomes at initiation.

In the same issue of Proc. US Nat. Acad. Sci. (page 65l), Colombo, Vesco and Baglioni report that the presence of ribosomal subunits may be necessary for the initiation of protein synthesis in mammalian cells as in bacteria and, moreover, that the dissociation of the $80 \mathrm{~S}$ ribosome may require energy. Their experiments were essentially to determine the changes in ribosome and subunit populations in reticulocytes and HeLa cells after blocking metabolism by the addition of $\mathrm{NaF}$ alone or with cyclohexamide, an inhibitor of protein synthesis. After treatment with NaF alone, the concentration of ribosome subunits decreases, indicating that free subunits are being used up at initiation and not reformed by the dissociation of free $80 S$ ribosomes. There is also a drop in the numbers of polysomes. The NaF apparently blocks dissociation of ribosomes but not initiation of protein synthesis or dissociation of polysomes. On the other hand, treatmont with cyclohexamide before treatment with $\mathrm{NaF}$ prevents the dissociation of polysomes and initiation of new chains, so the concentration of subunits remains more or less constant. On removal of $\mathrm{NaF}$, the reappearance of subunits precedes restoration of normal protein synthesis. These data support the notion that in eukaryotic cells there is a cyclical association and dissociation of ribosome subunits and that energy is probably required for the dissociation.

\section{HAEMOGLOBIN STRUCTURE \\ Dimers or Tetramers Again}

\section{from our Molecular Biology Correspondent}

THE mechanism of haem-haem interactions in haemoglobin, which give rise to the sigmoidal oxygen binding curve, has absorbed innumerable man-years of research: frequently during the past forty or so years the problem has seemed all but solved, only to surface again undiminished to fascinate and plague its devotees. Not long ago, the Benesches gave clear demonstration, strongly supported by the work of Guidotti, that interchange between dimer subunits occurs during oxygenation, and that the shape of the oxygen uptake curve can be described in terms of such a mechanism. This led to the hypothesis that the functional entity in haemoglobin is the symmetrical dimer.

The generality of this notion has been challenged by some kinetic experiments reported by Gibson and Parkhurst (J. Biol. Chem., 243, 5521; 1968) on the reaction of haemoglobin with carbon monoxide. Exposure of carboxyhaemoglobin to a high-intensity light flash causes the carbon monoxide to dissociate, with formation of a spectroscopically distinct product. This is "rapid" haemoglobin, presumed to be deoxyhaemoglobin which has not had time to revert to the characteristic conformation of this species at equilibrium. When deoxyhaemoglobin is then allowed to react with carbon monoxide, and the mixture is exposed to a flash at various reaction times (on a msec time- 